\title{
Considerações hidrodinâmicas sobre a derivação liquórica V: alterações nos dimensionamentos originais do sistema valvular no momento do implante alteram suas características hidrodinâmicas e induzem a erros de avaliação clínica pelo neurocirurgião
}

\author{
Ítalo Capraro Suriano', Ângelo Luiz Maset ${ }^{2}$, Thamires Arruda Fontolan ${ }^{3}$, \\ Ruy Monteiro4, José Ricardo Camilo Pinto ${ }^{5}$, José Ricardo Andrade', \\ Diogo Gonçalves Freitas 7 , Thiago Pereira Rodrigues ${ }^{8}$, Daniel Araújo Paz ${ }^{8}$, Sérgio Cavalheiro ${ }^{9}$
}

Departamento de Pesquisa e Desenvolvimento da Ventura Biomédica - Laboratório de Hidrodinâmica, São José do Rio Preto, SP, Brasil.

\section{RESUMO}

Objetivo: Averiguar se as características hidrodinâmicas das válvulas se alteram significativamente quando os dimensionamentos originais são violados e a possível repercussão clínica desse fato. Métodos: Foi utilizada uma bancada de testes automatizada, conforme a norma ISO 7197. Um sistema valvular foi testado inicialmente com os componentes preconizados e embalados originalmente com o produto. A seguir, substituiu-se o cateter peritoneal original por diversos dimensionamentos encontrados no mercado nacional. Nos testes para a avaliação da influência do diâmetro do cateter no escoamento, foi utilizado sempre o mesmo comprimento original do cateter. Aplicou-se o mesmo raciocínio lógico para a análise do comprimento do cateter. Nove perfis hidrodinâmicos foram obtidos para as diferentes montagens valvulares. Resultados: Diminuições imperceptíveis no diâmetro do cateter peritoneal alteraram o perfil hidrodinâmico do sistema valvular testado. Alterações de 0,1 $\mathrm{mm}$ no diâmetro de um cateter peritoneal aumentaram o efeito resistivo do sistema valvular suficientemente para que ele funcionasse fora do perfil hidrodinâmico preconizado; o efeito resistivo se acentuou conforme o diâmetro diminuiu, e vice-versa. A diminuição do comprimento do cateter também influenciou significativamente no desempenho hidráulico da válvula, de um regime inicial de alta pressão para um regime de média pressão. Conclusão: Alterações de dimensionamento em cateteres peritoneais trazem complicações para o paciente e desorientam o julgamento clínico do neurocirurgião no período pós-implante, pois o sistema valvular funcionará em regime hidráulico fora do especificado, e o raciocínio lógico do neurocirurgião também se torna comprometido. Esse fato pode ser crítico principalmente em crianças e neonatos. Não foram identificados relatos desse fato na literatura que antecede esta apresentação.

\section{PALAVRAS-CHAVE}

Hidrocefalia, derivação ventriculoperitoneal, síndrome do ventrículo fendido, hidrodinâmica.

\footnotetext{
Neurocirurgião, coordenador do PSNcr HSP da Universidade Federal de São Paulo (Unifesp), São Paulo, SP, Brasil. Neurocirurgião da Sociedade Beneficência Portuguesa de São José do Rio Preto, São José do Rio Preto, SP, Brasil.

Física biológica do Departamento de Hidrodinâmica de Ventura Biomédica Ltda., São José do Rio Preto, SP, Brasil.

Neurocirurgião, chefe de Serviço do Hospital Municipal Miguel Couto, Rio de Janeiro, RJ, Brasil.

Engenheiro mecânico do Departamento de Hidrodinâmica de Ventura Biomédica Ltda., São José do Rio Preto, SP, Brasil.

Técnico do Departamento de Hidrodinâmica de Ventura Biomédica Ltda., São José do Rio Preto, SP, Brasil.

Médico-residente do Hospital Municipal Miguel Couto, Rio de Janeiro, RJ, Brasil.

Médico-residente da Escola Paulista de Medicina da Unifesp, São Paulo, SP, Brasil.

9 Professor titular de Neurocirurgia da Escola Paulista de Medicina da Unifesp, São Paulo, SP, Brasil.
} 


\begin{abstract}
Modifications in catheter dimensions upon shunt implantation - Revision affect shunt hydrodynamic profile and clinical judgment

Objective: Check if the hydrodynamic characteristics of the valves change significantly when the original sizing are violated, and the possible clinical consequences of this fact. Methods: One shunt system was randomly submitted to hydraulic forces in a bench test according to ISO 7197, for 50, 40, 30, 20, 10 and $5 \mathrm{ml} / \mathrm{h}$ flow, in six sequential tests, and each sequential test was repeated 7 times. Results are the mean flow for the 7 events. Maintaining original peritoneal length of 1,200 $\mathrm{mm}$, data were collected for i.d. catheter diameters of 1,5, 1,2, 1,1, and 1,0 mm. Maintaining original i.d., peritoneal catheter length was sequentially cut and tested every $10 \mathrm{~cm}$ down to $600 \mathrm{~mm}$. Results: Changes in the order of $0,1 \mathrm{~mm}$ in catheter inner diameter changes the hydrodynamic profile of the shunt system. Cutting a peritoneal catheter by also changes its original hydrodynamic profile. Shunt will perform in a lower pressure setting from originally specified. Conclusion: Cutting peritoneal catheters upon implantation or revisions is a fact, especially in pediatric patients. Depending on the cutting length of the peritoneal catheter, the shunt system implanted will not perform according the its specifications, being a lower resistance shunt than preconized by the manufacturer, and the neurosurgeon may have misjudgments whenever the patient requires care for shunt malfunction.
\end{abstract}

\title{
KEYWORDS
}

Hydrocephalus, ventriculoperitoneal shunt, slit ventricle syndrome, hydrodynamics.

\section{Introdução}

Em nosso artigo anterior, ${ }^{1}$ demonstramos que todo sistema valvular funciona fisicamente como um conjunto de resistências em série, e cada componente opõe alguma resistência ao fluxo liquórico; a pressão da válvula, expressão conhecida pelo neurocirurgião, é a resultante (ou somatório) das resistências de todos os componentes do sistema valvular. Naquela publicação, também esclarecemos os critérios para a determinação da resistência a ser imposta pelo sistema valvular, que são determinados pela ISO 7197 , edição revisada de 2006, denominada Neurosurgical implants - Sterile, single-use hydrocephalus shunts and components. Esses critérios são seguidos pela maioria das indústrias.

É fato que os sistemas valvulares são fornecidos pela indústria médica com cateteres longos, em geral de 100 ou $120 \mathrm{~cm}$ de comprimento. Também é fato que revisões de válvulas são procedimentos comuns em neurocirurgia e, mais comumente, todo o sistema é substituído. Mas existem situações em que somente um cateter é substituído, assim como o corte do cateter peritoneal na população pediátrica e neonatal no momento do implante também é uma prática comum. Nesses casos, se determinado cateter for trocado por um outro mais longo ou mais curto, ou se determinado cateter sofrer alterações em seu diâmetro interno, o desempenho do sistema valvular poderá sofrer modificações e, portanto, o sistema valvular teoricamente não trabalhará com o perfil hidrodinâmico preconizado; as alterações hidrodinâmicas serão imperceptíveis e o julgamento clínico do neurocirurgião estará comprometido se o sistema valvular não tiver o desempenho hidrodinâmico esperado pelo neurocirurgião. Nesse contexto, foram exploradas as variáveis "raio" e "comprimento" de cateteres peritoneais em sistemas de válvulas para hidrocefalia, tendo como referencial inicial os componentes originais do sistema; se o sistema valvular sofrer interferência como demonstrado na equação de Poiseuille, isto é, se a resistência do sistema valvular se alterar inversamente à quarta potência do raio do tubo e/ou diretamente na mesma proporção ao comprimento do tubo, qual o efeito quando se alteram indiscriminadamente as características originais de um sistema valvular? As válvulas trabalham dentro das características hidrodinâmicas preconizadas pelo fabricante? O erro induzido pela alteração de um componente não original é negligível ou substancial? O objetivo deste trabalho é averiguar se as características hidrodinâmicas das válvulas se alteram quando os dimensionamentos originais são violados e analisar possível repercussão clínica desse fato.

\section{Materiais e métodos}

\section{A bancada de testes}

Para a realização dos ensaios hidrodinâmicos, foi utilizada uma bancada de testes automatizada, conforme a norma ISO 7197, já descrita detalhadamente em publicações anteriores. ${ }^{1-10}$ A bancada (Figura 1) é composta por um reservatório de vidro com água bidestilada à temperatura de $37 \pm 2{ }^{\circ} \mathrm{C}(\mathrm{B})$, controlada por um aquecedor programável $(\mathrm{C})$ sincronizado com um termômetro submerso (F). É utilizada uma bomba de infusão programável linear $(\mathrm{A})$, onde a água aquecida a aproximadamente $37 \pm 2{ }^{\circ} \mathrm{C}$ é bombeada por uma seringa de $60 \mathrm{ml}$ de extrema precisão até uma das vias da torneira de um tubo graduado (D), que determina a resistência diante das vazões de 50, 40, 30, 20, 10 e $5 \mathrm{ml} / \mathrm{h}$, respectivamente, em sete testes sequenciais 
com duração de aproximadamente $3 \mathrm{~min}$. Cada teste sequencial foi repetido por sete vezes, e os resultados representam a média dos sete eventos.

No presente trabalho, foi testada uma única válvula comercial modelo Synchrony - Cello, coletada aleatoriamente dos estoques da empresa Ventura Biomédica. O modelo selecionado foi uma válvula de alta pressão adulta, lote AUTO 080904, e número de série 7097, fabricada em fevereiro/2011. O sistema valvular foi testado inicialmente com os componentes preconizados e embalados originalmente com o produto, e o comprimento do cateter peritoneal possui $1.200 \mathrm{~mm}$ e diâmetro de $1,2 \mathrm{~mm}$. A seguir, substituiu-se o cateter peritoneal original pelos dimensionamentos mais comumente encontrados no mercado nacional. No total, foram utilizados os diâmetros de $1,5 \mathrm{~mm}, 1,2 \mathrm{~mm}, 1,1 \mathrm{~mm}$ e $1,0 \mathrm{~mm}$. Nos testes para a avaliação da influência do diâmetro do cateter no escoamento, foi utilizado sempre o mesmo comprimento original do cateter de $1.200 \mathrm{~mm}$. Finalizada a análise hidrodinâmica com as alterações no diâmetro, foram repetidos os testes, alterando-se agora apenas o comprimento do cateter. Manteve-se o diâmetro interno original de $1,2 \mathrm{~mm}$ e utilizaram-se sequencialmente $1.200,1.100,1.000,900,800$ e $600 \mathrm{~mm}$ de comprimento. Nove perfis hidrodinâmicos foram obtidos para as diferentes montagens de sistemas valvulares. Para maior clareza, as frações decimais foram descartadas.

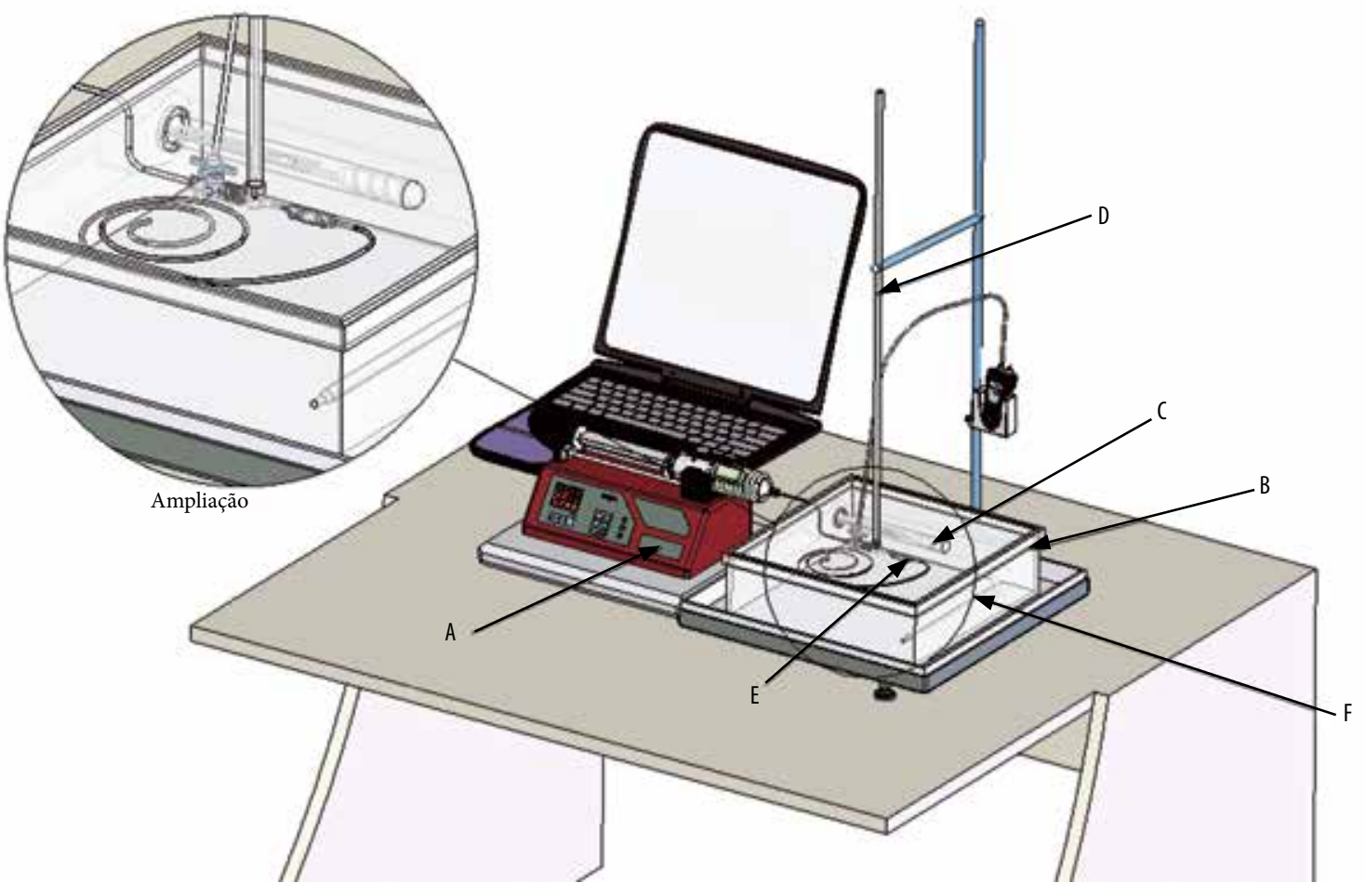

Figura 1 - Bancada testes hidrodinâmicos.

(A) Bomba de infusão, (B) Reservatório, (C) Termostato, (D) Manômetro graduado, (E) Válvula com cateter, (F) Termômetro.

\section{Resultado}

\section{Variação do diâmetro do cateter}

Mantendo-se o comprimento do cateter peritoneal constante em $1.200 \mathrm{~mm}$, os resultados obtidos experimentalmente com a variação do diâmetro do cateter estão sumarizados na tabela 1.

A média dos valores das pressões e respectivos desvios-padrões acima foram plotados no gráfico de perfil hidrodinâmico. A figura $2 \mathrm{~A}$ representa a faixa de tolerância do perfil hidrodinâmico para uma válvula de alta pressão preconizado pelo fabricante. A média pressórica para a vazão de $5 \mathrm{ml} / \mathrm{h}$ foi de $82 \mathrm{mmH}_{2} \mathrm{O}$ (desvio-padrão 11); a média pressórica para a vazão de $10 \mathrm{ml} / \mathrm{h}$ foi de $101 \mathrm{mmH}_{2} \mathrm{O}$ (desvio-padrão 7); a média pressórica para a vazão de $20 \mathrm{ml} / \mathrm{h}$ foi de $134 \mathrm{mmH}_{2} \mathrm{O}$ (desvio-padrão 6); a média pressórica para a vazão de $30 \mathrm{ml} / \mathrm{h}$ foi de 166 $\mathrm{mmH}_{2} \mathrm{O}$ (desvio-padrão 7); a média pressórica para a vazão de $40 \mathrm{ml} / \mathrm{h}$ foi de $192 \mathrm{mmH}_{2} \mathrm{O}$ (desvio-padrão 6); e a média pressórica para a vazão de $50 \mathrm{ml} / \mathrm{h}$ foi de 214 $\mathrm{mmH}_{2} \mathrm{O}$ (desvio-padrão 7). O sistema valvular testado funcionou dentro da faixa de tolerância e está representado pela linha tracejada com os respectivos desvios-pa- 
drões em cada nível de vazão testada. Os valores de cada teste estão disponibilizados no bloco II da tabela 1 .

A figura 2B representa o desempenho do sistema valvular quando utilizado um cateter peritoneal com um diâmetro interno maior, de $1,5 \mathrm{~mm}$. A média pressórica para a vazão de $5 \mathrm{ml} / \mathrm{h}$ foi de $47 \mathrm{mmH}_{2} \mathrm{O}$ (desvio-padrão 6); a média pressórica para a vazão de $10 \mathrm{ml} / \mathrm{h}$ foi de 65 $\mathrm{mmH}_{2} \mathrm{O}$ (desvio-padrão 6); a média pressórica para a vazão de $20 \mathrm{ml} / \mathrm{h}$ foi de $86 \mathrm{mmH}_{2} \mathrm{O}$ (desvio-padrão 9); a média pressórica para a vazão de $30 \mathrm{ml} / \mathrm{h}$ foi de 112 $\mathrm{mmH}_{2} \mathrm{O}$ (desvio-padrão 12); a média pressórica para a vazão de $40 \mathrm{ml} / \mathrm{h}$ foi de $132 \mathrm{mmH}_{2} \mathrm{O}$ (desvio-padrão 10); e a média pressórica para a vazão de $50 \mathrm{ml} / \mathrm{h}$ foi de 150 $\mathrm{mmH}_{2} \mathrm{O}$ (desvio-padrão 10). $\mathrm{O}$ sistema valvular testado apresentou menor resistência hidrodinâmica, totalmente fora dos limites aceitáveis como um sistema valvular de alta pressão. As médias e desvios-padrões para cada vazão estão disponibilizados no bloco I da tabela 1 .

\begin{tabular}{|c|c|c|c|c|c|c|c|c|c|}
\hline \multicolumn{10}{|l|}{ Bloco I } \\
\hline \multicolumn{10}{|c|}{ Cateter com o $\varnothing 1,5 \mathrm{DI}$} \\
\hline \multirow[t]{2}{*}{ Vazão } & \multicolumn{7}{|c|}{ Pressão $\mathrm{mmH}_{2} \mathrm{O}$} & Média & Desvio-padrão \\
\hline & I & II & III & IV & $\mathrm{V}$ & VI & VII & & \\
\hline $50 \mathrm{ml} / \mathrm{h}$ & 156 & 168 & 145 & 140 & 141 & 152 & 146 & 150 & 10 \\
\hline $40 \mathrm{ml} / \mathrm{h}$ & 144 & 145 & 132 & 132 & 119 & 131 & 119 & 132 & 10 \\
\hline $30 \mathrm{ml} / \mathrm{h}$ & 122 & 120 & 92 & 115 & 104 & 125 & 108 & 112 & 12 \\
\hline $20 \mathrm{ml} / \mathrm{h}$ & 95 & 89 & 86 & 94 & 73 & 92 & 75 & 86 & 9 \\
\hline $10 \mathrm{ml} / \mathrm{h}$ & 65 & 60 & 67 & 73 & 69 & 66 & 57 & 65 & 6 \\
\hline $5 \mathrm{ml} / \mathrm{h}$ & 47 & 45 & 43 & 54 & 55 & 40 & 43 & 47 & 6 \\
\hline \multicolumn{10}{|l|}{ Bloco II } \\
\hline \multicolumn{10}{|c|}{ Cateter com $\emptyset 1,2 \mathrm{~mm}$ DI } \\
\hline \multirow[t]{2}{*}{ Vazão } & \multicolumn{7}{|c|}{ Pressão $\mathrm{mmH}_{2} \mathrm{O}$} & Média & Desvio-padrão \\
\hline & I & II & III & IV & $\mathrm{V}$ & VI & VII & & \\
\hline $50 \mathrm{ml} / \mathrm{h}$ & 220 & 221 & 217 & 216 & 204 & 206 & 215 & 214 & 7 \\
\hline $40 \mathrm{ml} / \mathrm{h}$ & 191 & 197 & 193 & 196 & 179 & 190 & 195 & 192 & 6 \\
\hline $30 \mathrm{ml} / \mathrm{h}$ & 162 & 165 & 173 & 172 & 161 & 157 & 173 & 166 & 7 \\
\hline $20 \mathrm{ml} / \mathrm{h}$ & 139 & 135 & 135 & 132 & 128 & 125 & 142 & 134 & 6 \\
\hline $10 \mathrm{ml} / \mathrm{h}$ & 101 & 95 & 100 & 106 & 102 & 90 & 113 & 101 & 7 \\
\hline $5 \mathrm{ml} / \mathrm{h}$ & 87 & 81 & 73 & 76 & 82 & 70 & 104 & 82 & 11 \\
\hline \multicolumn{10}{|c|}{ Bloco III } \\
\hline \multicolumn{10}{|c|}{ Cateter com $\emptyset 1,1 \mathrm{~mm}$ DI } \\
\hline \multirow[t]{2}{*}{ Vazão } & \multicolumn{7}{|c|}{ Pressão $\mathrm{mmH}_{2} \mathrm{O}$} & Média & Desvio-padrão \\
\hline & I & II & III & IV & $\mathrm{V}$ & VI & VII & & \\
\hline $50 \mathrm{ml} / \mathrm{h}$ & 240 & 232 & 230 & 223 & 221 & 228 & 230 & 229 & 6 \\
\hline $40 \mathrm{ml} / \mathrm{h}$ & 211 & 213 & 210 & 199 & 197 & 202 & 205 & 205 & 6 \\
\hline $30 \mathrm{ml} / \mathrm{h}$ & 189 & 193 & 195 & 180 & 181 & 185 & 187 & 187 & 6 \\
\hline $20 \mathrm{ml} / \mathrm{h}$ & 152 & 150 & 157 & 153 & 168 & 154 & 163 & 157 & 7 \\
\hline $10 \mathrm{ml} / \mathrm{h}$ & 110 & 105 & 120 & 151 & 135 & 128 & 130 & 126 & 16 \\
\hline $5 \mathrm{ml} / \mathrm{h}$ & 83 & 85 & 90 & 120 & 101 & 95 & 105 & 97 & 13 \\
\hline \multicolumn{10}{|l|}{ Bloco IV } \\
\hline \multicolumn{10}{|c|}{ Cateter com $\varnothing 1,0$ mm DI } \\
\hline \multirow[t]{2}{*}{ Vazão } & \multicolumn{7}{|c|}{ Pressão $\mathrm{mmH}_{2} \mathrm{O}$} & Média & Desvio-padrão \\
\hline & I & II & III & IV & $\mathrm{V}$ & VI & VII & & \\
\hline $50 \mathrm{ml} / \mathrm{h}$ & 240 & 238 & 241 & 236 & 240 & 247 & 240 & 240 & 3 \\
\hline $40 \mathrm{ml} / \mathrm{h}$ & 226 & 223 & 224 & 220 & 228 & 224 & 201 & 221 & 9 \\
\hline $30 \mathrm{ml} / \mathrm{h}$ & 212 & 206 & 206 & 204 & 212 & 214 & 193 & 207 & 7 \\
\hline $20 \mathrm{ml} / \mathrm{h}$ & 175 & 187 & 173 & 172 & 178 & 168 & 169 & 175 & 6 \\
\hline $10 \mathrm{ml} / \mathrm{h}$ & 139 & 155 & 139 & 148 & 138 & 149 & 145 & 145 & 6 \\
\hline $5 \mathrm{ml} / \mathrm{h}$ & 110 & 116 & 116 & 108 & 118 & 113 & 113 & 113 & 4 \\
\hline
\end{tabular}


A figura $2 \mathrm{C}$ representa o desempenho do sistema valvular quando utilizado um cateter peritoneal com um diâmetro interno menor do que o preconizado pelo fabricante, de $1,1 \mathrm{~mm}$. A média pressórica para a vazão de $5 \mathrm{ml} / \mathrm{h}$ foi de $97 \mathrm{mmH}_{2} \mathrm{O}$ (desvio-padrão 13); a média pressórica para a vazão de $10 \mathrm{ml} / \mathrm{h}$ foi de $126 \mathrm{mmH}_{2} \mathrm{O}$ (desvio-padrão 16); a média pressórica para a vazão de $20 \mathrm{ml} / \mathrm{h}$ foi de $157 \mathrm{mmH}_{2} \mathrm{O}$ (desvio-padrão 7); a média pressórica para a vazão de $30 \mathrm{ml} / \mathrm{h}$ foi de $187 \mathrm{mmH}_{2} \mathrm{O}$ (desvio-padrão 6); a média pressórica para a vazão de $40 \mathrm{ml} / \mathrm{h}$ foi de 205 $\mathrm{mmH}_{2} \mathrm{O}$ (desvio-padrão 6); e a média pressórica para a vazão de $50 \mathrm{ml} / \mathrm{h}$ foi de $229 \mathrm{mmH}_{2} \mathrm{O}$ (desvio-padrão 6). Com o diâmetro interno apenas $0,1 \mathrm{~mm}$ menor, o sistema valvular testado apresentou maior resistência hidrodinâmica, já fora dos limites aceitáveis como uma válvula de alta pressão. As médias e desvios-padrões para cada vazão estão disponibilizados no bloco III da tabela 1 .
A figura 2D representa o desempenho do sistema valvular quando se utiliza um cateter peritoneal com um diâmetro interno ainda menor que o preconizado pelo fabricante, de $1,0 \mathrm{~mm}$. A média pressórica para a vazão de $5 \mathrm{ml} / \mathrm{h}$ foi de $113 \mathrm{mmH}_{2} \mathrm{O}$ (desvio-padrão 4); a média pressórica para a vazão de $10 \mathrm{ml} / \mathrm{h}$ foi de 145 $\mathrm{mmH}_{2} \mathrm{O}$ (desvio-padrão 6); a média pressórica para a vazão de $20 \mathrm{ml} / \mathrm{h}$ foi de $175 \mathrm{mmH}_{2} \mathrm{O}$ (desvio-padrão 6); a média pressórica para a vazão de $30 \mathrm{ml} / \mathrm{h}$ foi de 207 $\mathrm{mmH}_{2} \mathrm{O}$ (desvio-padrão 7); a média pressórica para a vazão de $40 \mathrm{ml} / \mathrm{h}$ foi de $221 \mathrm{mmH}_{2} \mathrm{O}$ (desvio-padrão 9); e a média pressórica para a vazão de $50 \mathrm{ml} / \mathrm{h}$ foi de $240 \mathrm{mmH}_{2} \mathrm{O}$ (desvio-padrão 3). Com apenas 0,2 $\mathrm{mm}$ de diferença com relação ao diâmetro original do produto $(1,2 \mathrm{~mm})$, o sistema valvular testado apresentou uma resistência hidrodinâmica muito acima das especificações da válvula de alta pressão. As médias e desvios-padrões para cada vazão estão disponibilizados no bloco IV da tabela 1.

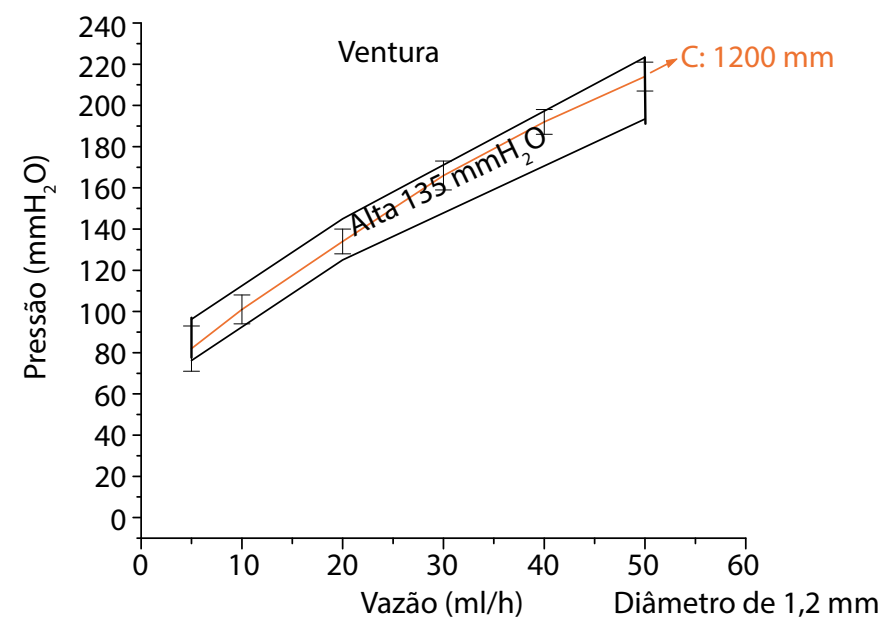

Figura $2 \mathrm{~A}$ - O sistema valvular (SV) com os componentes diâmetro interno originais.

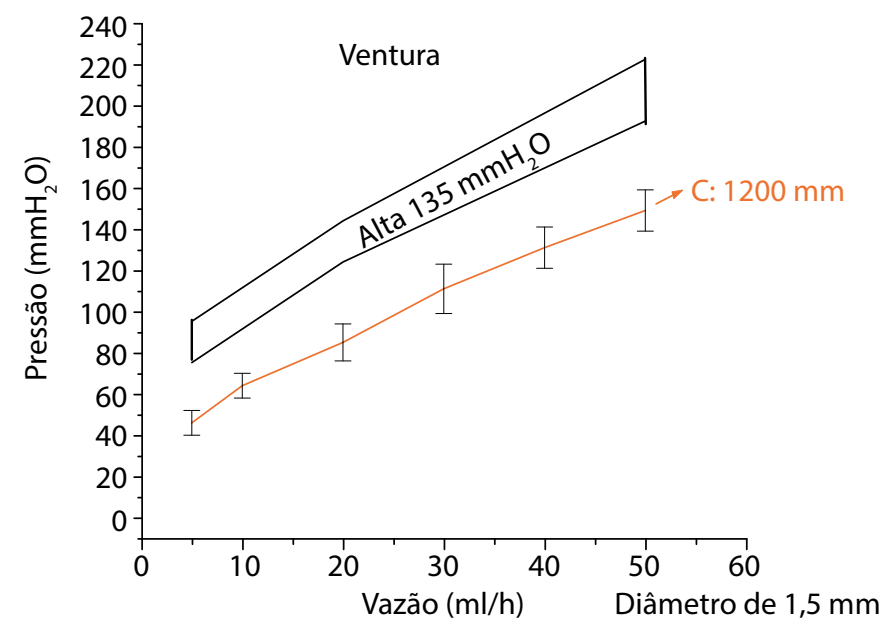

Figura 2B - SV com o cateter peritoneal de 0,3 mm maior. 


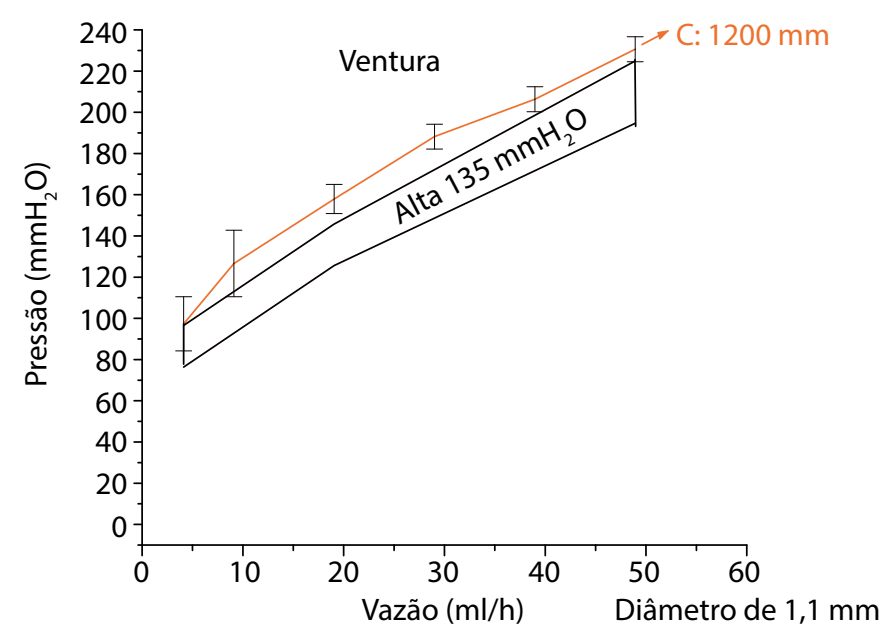

Figura $2 C-S V$ com o cateter peritoneal com diâmetro interno 0,1 mm menor.

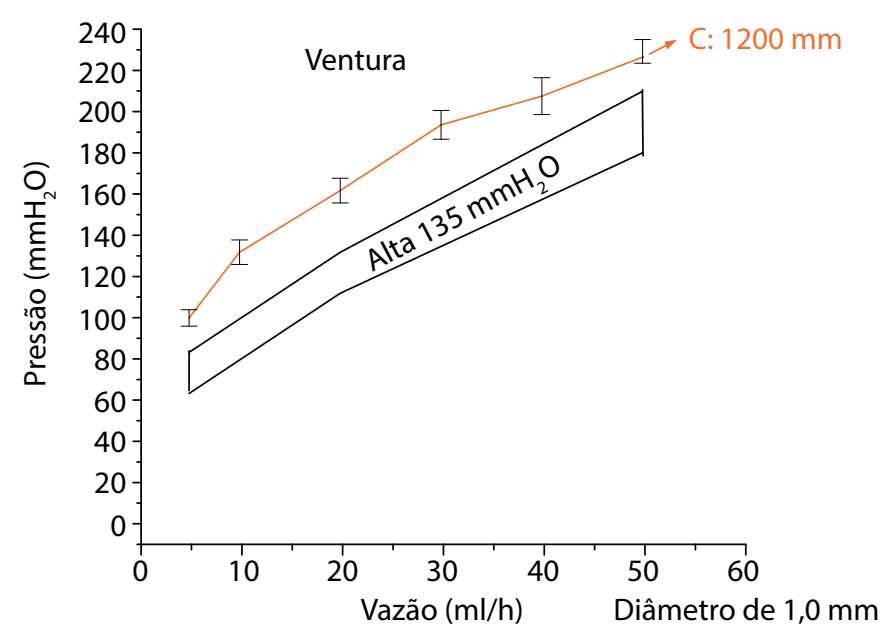

Figura $2 D-S V$ com o cateter peritoneal com diâmetro interno 0,2 mm menor.

\section{Variação do comprimento do cateter}

Mantendo-se o diâmetro do cateter peritoneal constante em 1,2 mm, os resultados obtidos experimentalmente com a variação do comprimento estão sumarizados na tabela 2. A média dos valores das pressões e respectivos desvios-padrões da tabela 2 foram plotados no gráfico de perfil hidrodinâmico.

A figura $3 \mathrm{~A}$ representa a faixa de tolerância do perfil hidrodinâmico para um sistema valvular de alta pressão preconizada pelo fabricante, exatamente o mesmo bloco II da tabela 1 (Figura 1A), reinserida para melhor visualização. A figura 3B representa o desempenho do sistema valvular quando utilizado um cateter peritoneal com o comprimento de $1.100 \mathrm{~mm}$ (ou seja, foram cortados $10 \mathrm{~cm}$ do comprimento preconizado pelo fabricante). A média pressórica para a vazão de $5 \mathrm{ml} / \mathrm{h}$ foi de 88 $\mathrm{mmH}_{2} \mathrm{O}$ (desvio-padrão 3); a média pressórica para a vazão de $10 \mathrm{ml} / \mathrm{h}$ foi de $101 \mathrm{mmH}_{2} \mathrm{O}$ (desvio-padrão 6); a média pressórica para a vazão de $20 \mathrm{ml} / \mathrm{h}$ foi de 130 $\mathrm{mmH}_{2} \mathrm{O}$ (desvio-padrão 4); a média pressórica para a vazão de $30 \mathrm{ml} / \mathrm{h}$ foi de $150 \mathrm{mmH}_{2} \mathrm{O}$ (desvio-padrão 2); a média pressórica para a vazão de $40 \mathrm{ml} / \mathrm{h}$ foi de 181 $\mathrm{mmH}_{2} \mathrm{O}$ (desvio-padrão 6); e a média pressórica para a vazão de $50 \mathrm{ml} / \mathrm{h}$ foi de $199 \mathrm{mmH}_{2} \mathrm{O}$ (desvio-padrão 8). O sistema valvular testado apresentou uma menor resistência à drenagem do fluido, mas funcionou dentro da faixa de tolerância. Os valores estão disponibilizados no bloco II da tabela 2. 
Tabela 2 -Diâmetro de 1,2 mm

Bloco I

Tabela $2-$ Diametro de 1,2 mim

\begin{tabular}{|c|c|c|c|c|c|c|c|c|c|}
\hline \multicolumn{10}{|c|}{ Cateter com comprimento $1.200 \mathrm{~mm}$} \\
\hline \multirow[t]{2}{*}{ Vazão } & \multicolumn{7}{|c|}{ Pressão $\mathrm{mmH}_{2} \mathrm{O}$} & \multirow[t]{2}{*}{ Média } & \multirow[t]{2}{*}{ Desvio-padrão } \\
\hline & I & II & III & IV & $\mathrm{V}$ & VI & VII & & \\
\hline $50 \mathrm{ml} / \mathrm{h}$ & 220 & 221 & 217 & 216 & 204 & 206 & 215 & 214 & 7 \\
\hline $40 \mathrm{ml} / \mathrm{h}$ & 191 & 197 & 193 & 196 & 179 & 190 & 195 & 192 & 6 \\
\hline $30 \mathrm{ml} / \mathrm{h}$ & 162 & 165 & 173 & 172 & 161 & 157 & 173 & 166 & 7 \\
\hline $20 \mathrm{ml} / \mathrm{h}$ & 139 & 135 & 135 & 132 & 128 & 125 & 142 & 134 & 6 \\
\hline $10 \mathrm{ml} / \mathrm{h}$ & 101 & 95 & 100 & 106 & 102 & 90 & 113 & 101 & 7 \\
\hline $5 \mathrm{ml} / \mathrm{h}$ & 87 & 81 & 73 & 76 & 82 & 70 & 104 & 82 & 11 \\
\hline \multicolumn{10}{|l|}{ Bloco II } \\
\hline \multicolumn{10}{|c|}{ Cateter com comprimento de $1.100 \mathrm{~mm}$} \\
\hline \multirow[t]{2}{*}{ Vazão } & \multicolumn{7}{|c|}{ Pressão $\mathrm{mmH}_{2} \mathrm{O}$} & Média & Desvio-padrão \\
\hline & I & II & III & IV & $\mathrm{V}$ & VI & VII & & \\
\hline $50 \mathrm{ml} / \mathrm{h}$ & 198 & 195 & 193 & 209 & 192 & 211 & 197 & 199 & 8 \\
\hline $40 \mathrm{ml} / \mathrm{h}$ & 178 & 174 & 177 & 191 & 178 & 189 & 179 & 181 & 6 \\
\hline $30 \mathrm{ml} / \mathrm{h}$ & 147 & 150 & 149 & 152 & 150 & 151 & 150 & 150 & 2 \\
\hline $20 \mathrm{ml} / \mathrm{h}$ & 130 & 124 & 125 & 132 & 132 & 133 & 134 & 130 & 4 \\
\hline $10 \mathrm{ml} / \mathrm{h}$ & 98 & 96 & 103 & 102 & 111 & 102 & 92 & 101 & 6 \\
\hline $5 \mathrm{ml} / \mathrm{h}$ & 82 & 86 & 92 & 90 & 91 & 89 & 87 & 88 & 3 \\
\hline \multicolumn{10}{|c|}{ Bloco III } \\
\hline \multicolumn{10}{|c|}{ Cateter com comprimento de $1.000 \mathrm{~mm}$} \\
\hline \multirow[t]{2}{*}{ Vazão } & \multicolumn{7}{|c|}{ Pressão $\mathrm{mmH}_{2} \mathrm{O}$} & Média & Desvio-padrão \\
\hline & I & II & III & IV & $\mathrm{V}$ & VI & VII & & \\
\hline $50 \mathrm{ml} / \mathrm{h}$ & 188 & 196 & 193 & 189 & 195 & 182 & 176 & 188 & 7 \\
\hline $40 \mathrm{ml} / \mathrm{h}$ & 170 & 177 & 175 & 170 & 179 & 165 & 153 & 170 & 9 \\
\hline $30 \mathrm{ml} / \mathrm{h}$ & 144 & 142 & 145 & 147 & 150 & 143 & 130 & 143 & 6 \\
\hline $20 \mathrm{ml} / \mathrm{h}$ & 122 & 120 & 118 & 116 & 114 & 105 & 101 & 114 & 8 \\
\hline $10 \mathrm{ml} / \mathrm{h}$ & 93 & 90 & 96 & 94 & 97 & 88 & 83 & 92 & 5 \\
\hline $5 \mathrm{ml} / \mathrm{h}$ & 72 & 75 & 73 & 71 & 77 & 65 & 62 & 71 & 5 \\
\hline \multicolumn{10}{|c|}{ Bloco IV } \\
\hline \multicolumn{10}{|c|}{ Cateter com comprimento de $900 \mathrm{~mm}$} \\
\hline Vazão & & & Press & $\mathrm{mH}_{2}$ & & & & Média & Desvio-padrão \\
\hline & I & II & III & IV & $\mathrm{V}$ & VI & VII & & \\
\hline $50 \mathrm{ml} / \mathrm{h}$ & 178 & 179 & 169 & 170 & 185 & 172 & 178 & 176 & 6 \\
\hline $40 \mathrm{ml} / \mathrm{h}$ & 162 & 163 & 164 & 158 & 160 & 158 & 157 & 160 & 3 \\
\hline $30 \mathrm{ml} / \mathrm{h}$ & 127 & 137 & 129 & 125 & 126 & 123 & 127 & 128 & 4 \\
\hline $20 \mathrm{ml} / \mathrm{h}$ & 109 & 105 & 103 & 107 & 108 & 109 & 104 & 106 & 2 \\
\hline $10 \mathrm{ml} / \mathrm{h}$ & 84 & 82 & 83 & 81 & 84 & 86 & 81 & 83 & 2 \\
\hline $5 \mathrm{ml} / \mathrm{h}$ & 71 & 67 & 65 & 65 & 67 & 71 & 69 & 68 & 3 \\
\hline Bloco V & & & & & & & & & \\
\hline Cateter c & & & & & & & & & \\
\hline Vazão & & & Press & $\mathrm{mH}_{2}$ & & & & Média & Desvio-padrão \\
\hline & I & II & III & IV & $\mathrm{V}$ & VI & VII & & \\
\hline $50 \mathrm{ml} / \mathrm{h}$ & 163 & 165 & 166 & 159 & 163 & 158 & 166 & 163 & 3 \\
\hline $40 \mathrm{ml} / \mathrm{h}$ & 150 & 154 & 153 & 152 & 150 & 148 & 150 & 151 & 2 \\
\hline $30 \mathrm{ml} / \mathrm{h}$ & 112 & 107 & 109 & 110 & 114 & 108 & 109 & 110 & 2 \\
\hline $20 \mathrm{ml} / \mathrm{h}$ & 89 & 92 & 91 & 87 & 89 & 92 & 86 & 89 & 2 \\
\hline $10 \mathrm{ml} / \mathrm{h}$ & 72 & 73 & 69 & 70 & 72 & 71 & 67 & 71 & 2 \\
\hline $5 \mathrm{ml} / \mathrm{h}$ & 61 & 64 & 55 & 62 & 59 & 62 & 62 & 61 & 3 \\
\hline Bloco V & & & & & & & & & \\
\hline Cateter $\mathrm{c}$ & & & & & & & & & \\
\hline Vazão & & & Press & $\mathrm{mH}_{2}$ & & & & Média & Desvio-padrão \\
\hline & I & II & III & IV & $\mathrm{V}$ & VI & VII & & \\
\hline $50 \mathrm{ml} / \mathrm{h}$ & 143 & 135 & 142 & 135 & 155 & 141 & 148 & 143 & 7 \\
\hline $40 \mathrm{ml} / \mathrm{h}$ & 125 & 117 & 122 & 120 & 132 & 126 & 124 & 124 & 5 \\
\hline $30 \mathrm{ml} / \mathrm{h}$ & 91 & 94 & 106 & 104 & 112 & 110 & 109 & 104 & 8 \\
\hline $20 \mathrm{ml} / \mathrm{h}$ & 70 & 75 & 84 & 89 & 90 & 87 & 89 & 83 & 8 \\
\hline $10 \mathrm{ml} / \mathrm{h}$ & 58 & 53 & 56 & 58 & 56 & 63 & 63 & 58 & 4 \\
\hline $5 \mathrm{ml} / \mathrm{h}$ & 46 & 40 & 38 & 42 & 41 & 50 & 53 & 44 & 6 \\
\hline
\end{tabular}




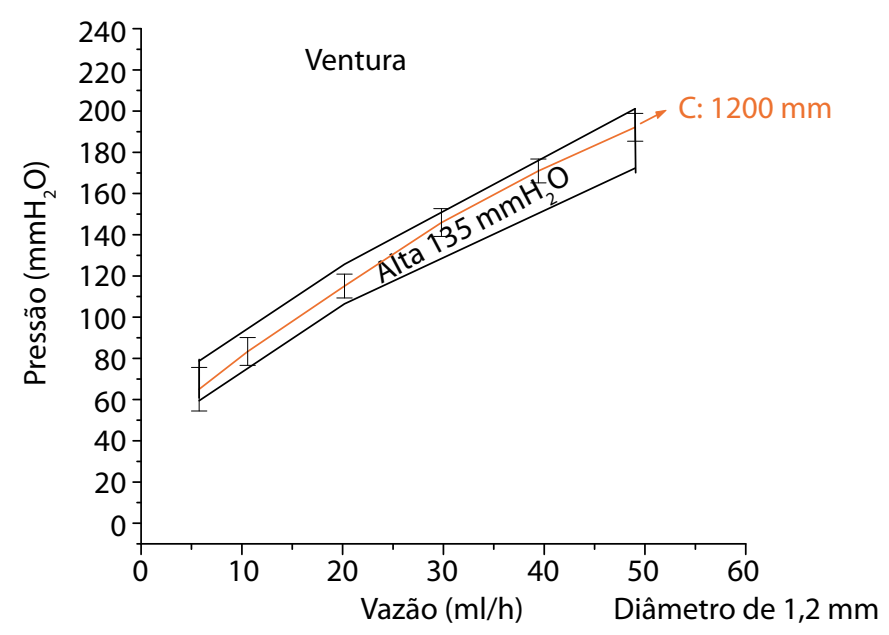

Figura 3A - Dimensionamento original.

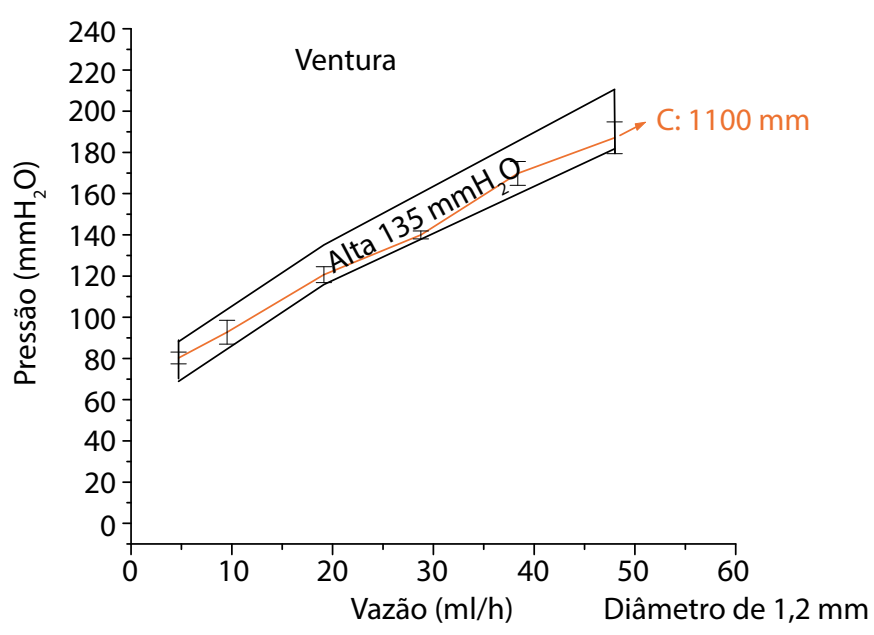

Figura 3B - Comprimento de $1.100 \mathrm{~mm}(-100 \mathrm{~mm})$.

A figura 3C representa o desempenho do sistema valvular quando utilizado um cateter peritoneal com o comprimento de $1.000 \mathrm{~mm}$ (ou seja, foram cortados $20 \mathrm{~cm}$ do comprimento preconizado pelo fabricante). A média pressórica para a vazão de $5 \mathrm{ml} / \mathrm{h}$ foi de 71 $\mathrm{mmH}_{2} \mathrm{O}$ (desvio-padrão 5); a média pressórica para a vazão de $10 \mathrm{ml} / \mathrm{h}$ foi de $92 \mathrm{mmH}_{2} \mathrm{O}$ (desvio-padrão 5); a média pressórica para a vazão de $20 \mathrm{ml} / \mathrm{h}$ foi de 114 $\mathrm{mmH}_{2} \mathrm{O}$ (desvio-padrão 8 ); a média pressórica para a vazão de $30 \mathrm{ml} / \mathrm{h}$ foi de $143 \mathrm{mmH}_{2} \mathrm{O}$ (desvio-padrão 6); a média pressórica para a vazão de $40 \mathrm{ml} / \mathrm{h}$ foi de 170 $\mathrm{mmH}_{2} \mathrm{O}$ (desvio-padrão 9); e a média pressórica para a vazão de $50 \mathrm{ml} / \mathrm{h}$ foi de $188 \mathrm{mmH}_{2} \mathrm{O}$ (desvio-padrão 7). O sistema valvular testado agora funcionou abaixo da faixa de tolerância. Os valores estão disponibilizados no bloco III da tabela 2 .
A figura 3D representa o desempenho do sistema valvular quando utilizado um cateter peritoneal com o comprimento de $900 \mathrm{~mm}$ (ou seja, foram cortados $30 \mathrm{~cm}$ do comprimento preconizado pelo fabricante). A média pressórica para a vazão de $5 \mathrm{ml} / \mathrm{h}$ foi de $68 \mathrm{mmH}_{2} \mathrm{O}$ (desvio-padrão 3); a média pressórica para a vazão de $10 \mathrm{ml} / \mathrm{h}$ foi de $83 \mathrm{mmH}_{2} \mathrm{O}$ (desvio-padrão 2); a média pressórica para a vazão de $20 \mathrm{ml} / \mathrm{h}$ foi de $106 \mathrm{mmH}_{2} \mathrm{O}$ (desvio-padrão 2); a média pressórica para a vazão de $30 \mathrm{ml} / \mathrm{h}$ foi de $128 \mathrm{mmH}_{2} \mathrm{O}$ (desvio-padrão 4); a média pressórica para a vazão de $40 \mathrm{ml} / \mathrm{h}$ foi de $160 \mathrm{mmH}_{2} \mathrm{O}$ (desvio-padrão 3); e a média pressórica para a vazão de $50 \mathrm{ml} / \mathrm{h}$ foi de $176 \mathrm{mmH}_{2} \mathrm{O}$ (desvio-padrão 6). O sistema valvular testado agora funcionou nitidamente abaixo da faixa de tolerância. Os valores estão disponibilizados no bloco IV da tabela 2. 
A figura 3E representa o desempenho do sistema valvular quando utilizado um cateter peritoneal com o comprimento de $800 \mathrm{~mm}$ (ou seja, foram cortados $40 \mathrm{~cm}$ do comprimento preconizado pelo fabricante). A média pressórica para a vazão de $5 \mathrm{ml} / \mathrm{h}$ foi de $61 \mathrm{mmH}_{2} \mathrm{O}$ (desvio-padrão 3); a média pressórica para a vazão de $10 \mathrm{ml} / \mathrm{h}$ foi de $71 \mathrm{mmH}_{2} \mathrm{O}$ (desvio-padrão 2); a média pressórica para a vazão de $20 \mathrm{ml} / \mathrm{h}$ foi de $89 \mathrm{mmH}_{2} \mathrm{O}$ (desvio-padrão 2); a média pressórica para a vazão de $30 \mathrm{ml} / \mathrm{h}$ foi de $110 \mathrm{mmH}_{2} \mathrm{O}$ (desvio-padrão 2); a média pressórica para a vazão de $40 \mathrm{ml} / \mathrm{h}$ foi de $151 \mathrm{mmH}_{2} \mathrm{O}$ (desvio-padrão 2); e a média pressórica para a vazão de $50 \mathrm{ml} / \mathrm{h}$ foi de $163 \mathrm{mmH}_{2} \mathrm{O}$ (desvio-padrão 3). O sistema valvular testado funcionou dentro da faixa de tolerância de uma outra categoria de válvulas do fabricante, conceituadas como sistema valvular de pressão normal. Os valores estão disponibilizados no bloco $\mathrm{V}$ da tabela 2.
A figura 3F representa o desempenho do sistema valvular quando utilizado um cateter peritoneal com o comprimento de $600 \mathrm{~mm}$ (ou seja, foram cortados $60 \mathrm{~cm}$ do comprimento preconizado pelo fabricante). A média pressórica para a vazão de $5 \mathrm{ml} / \mathrm{h}$ foi de 44 $\mathrm{mmH}_{2} \mathrm{O}$ (desvio-padrão 6); a média pressórica para a vazão de $10 \mathrm{ml} / \mathrm{h}$ foi de $58 \mathrm{mmH}_{2} \mathrm{O}$ (desvio-padrão 4); a média pressórica para a vazão de $20 \mathrm{ml} / \mathrm{h}$ foi de 83 $\mathrm{mmH}_{2} \mathrm{O}$ (desvio-padrão 8); a média pressórica para a vazão de $30 \mathrm{ml} / \mathrm{h}$ foi de $104 \mathrm{mmH}_{2} \mathrm{O}$ (desvio-padrão 8); a média pressórica para a vazão de $40 \mathrm{ml} / \mathrm{h}$ foi de $124 \mathrm{mmH}_{2} \mathrm{O}$ (desvio-padrão 5); e a média pressórica para a vazão de $50 \mathrm{ml} / \mathrm{h}$ foi de $143 \mathrm{mmH}_{2} \mathrm{O}$ (desvio -padrão 7). O sistema valvular testado funcionou dentro da faixa de tolerância de uma válvula de média pressão. Os valores estão disponibilizados no bloco VI da tabela 2.

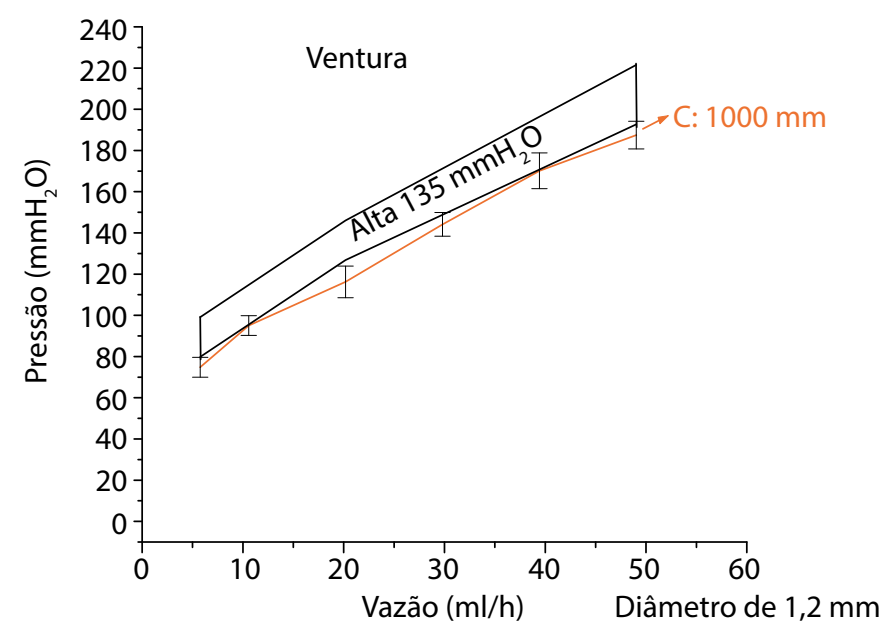

Figura 3C - Comprimento de $1.000 \mathrm{~mm}$ (- $200 \mathrm{~mm})$.

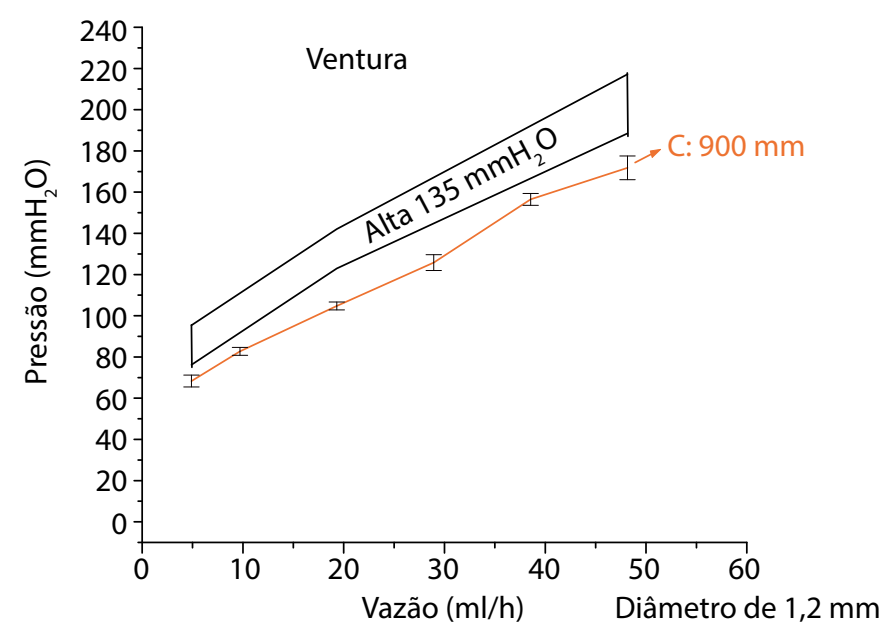

Figura 3D - Comprimento de $900 \mathrm{~mm}(-300 \mathrm{~mm})$. 


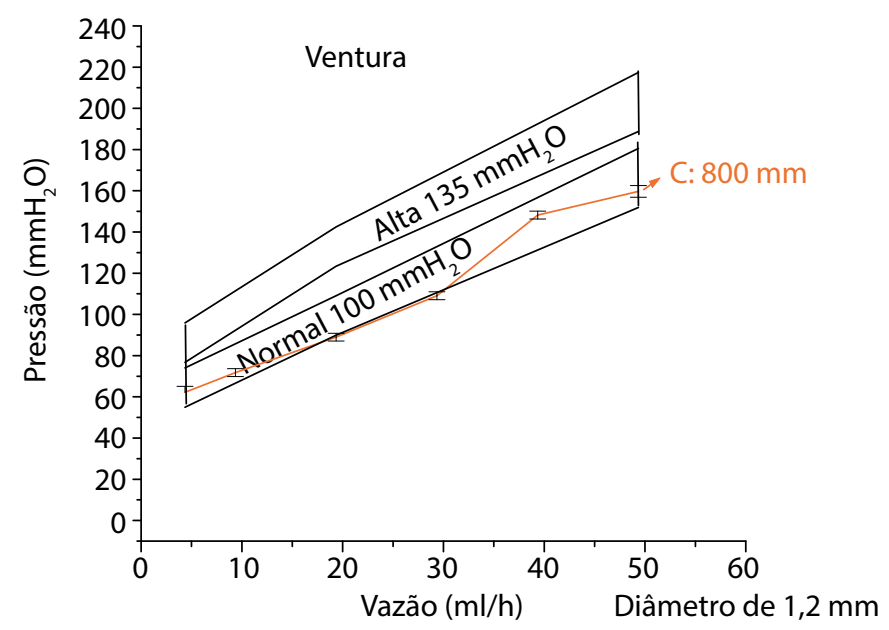

Figura 3E - Comprimento $800 \mathrm{~mm}(-400 \mathrm{~mm})$.

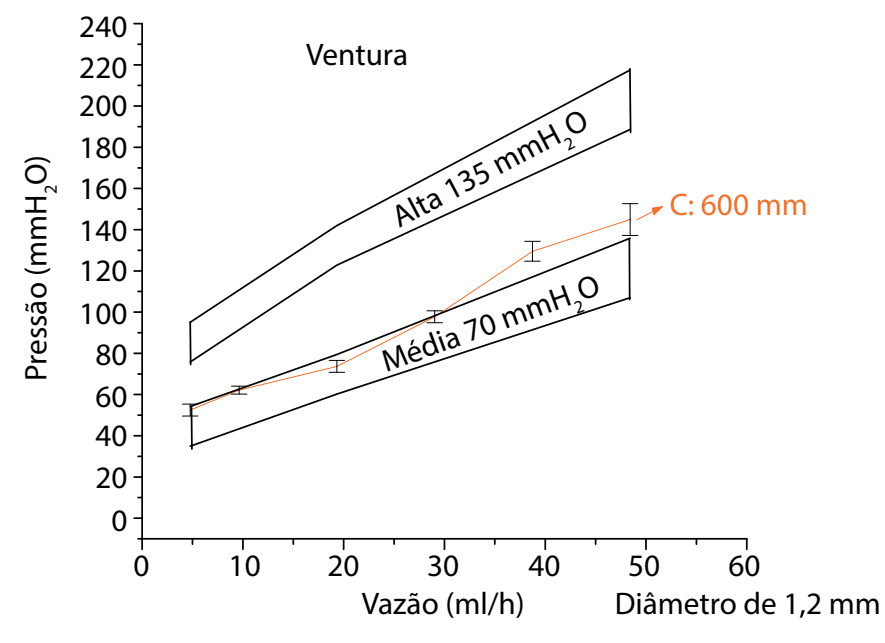

Figura 3F - Comprimento $600 \mathrm{~mm}$ (- $600 \mathrm{~mm})$.

\section{Discussão}

Distúrbios hidrostáticos e hidrodinâmicos, clinicamente se refletindo como hipodrenagem ou hiperdrenagem, são comuns seguindo-se ao implante de válvulas. ${ }^{11,12}$

O sistema nervoso como um todo trabalha em regimes pressóricos bastante baixos, suportando picos hipertensivos eventuais, e a derivação liquórica permanente (DVP) tenta reproduzir os regimes pressóricos fisiológicos. Existem inúmeros trabalhos que expressam a relação matemática das forças envolvidas nos sistemas valvulares, mas credita-se a Fox et al. ${ }^{13,14}$ como os primeiros a descreverem os fatores que influenciam a pressão de perfusão $(\Delta \mathrm{P})$ e fluxo $(\mathrm{F})$ por meio da seguinte relação:

$\Delta \mathrm{P}=\mathrm{PIC}+\mathrm{PH}-(\mathrm{PCD}+\mathrm{PF})$, em que $\Delta \mathrm{P}$ é a pressão de perfusão, PIC é a pressão ventricular, bPH é a pressão hidrostática do cateter distal, PCD é a pressão na cavidade distal e PF é a resistência imposta pelo sistema valvular.

O modelo de Fox enfatiza a importância da pressão hidrostática do cateter distal nos fenômenos de hiperdrenagem, mas não explica a hiperdrenagem em pacientes na posição horizontal. O grupo de De Jong et $a l .{ }^{15}$ explorou esse fato e propôs conceitos alternativos, em que as ondas B impulsionariam parte do LCR para a cavidade peritoneal ou atrial, causando esvaziamento do compartimento liquórico e aumento compensatório do volume sanguíneo cerebral por meio de dilatação venosa parenquimatosa com consequente exaustão da complacência cerebral. Quando o paciente assume a posição ereta, a influência hidrostática da coluna liquórica não pode ser equilibrada, ocorrendo diminuição repentina da pressão intraventricular e aumento da pressão transmural sobre a veia-ponte e lacuna, o que supostamente leva ao rompimento delas, causando, consequentemente, hematoma subdural. Portanto, o 
modelo apresentado por De Jong et al. ${ }^{15}$ enfatiza a responsabilidade coletiva da coluna hidrostática liquórica mas também a efetiva participação da elasticidade (ou complacência) cerebral como fatores de estabelecimento de um alto gradiente pressórico nos movimentos de verticalização entre as posições horizontal e ortostática. Uma outra conclusão do trabalho de De Jong foi a alta incidência de hematoma subdural em pacientes que tiveram sistemas valvulares com regime de baixa pressão implantados ( $71 \%$ contra $34 \%)$. Esses valores foram estatisticamente significantes para $\mathrm{p}<0,002$.

Quase simultaneamente, Kajimoto et al. ${ }^{16}$ demonstraram o efeito da pressão intra-abdominal nas variáveis do modelo descrito por Fox ${ }^{13,14} \mathrm{e}$ teorizaram que a relativa baixa incidência de hiperdrenagem ocorre devido ao efeito compensatório da pressão intra-abdominal sobre os efeitos da pressão hidrostática. Num artigo relativamente recente de Mukerji et al. ${ }^{17}$ três diferentes comprimentos de cateter utilizados comercialmente para pseudotumor cerebral foram avaliados sob diferentes simulações de pressão intraventricular e pressão intra-abdominal. Como seria esperado pelas leis físicas mencionadas logo a seguir, houve grande variação de fluxo entre diferentes comprimentos testados. Mukerji et al. ${ }^{17}$ concluíram que as soluções de cateteres atuais para procedimentos de derivação lombar são insatisfatórias e os índices de complicações atuais são inaceitáveis, sugerindo como solução imediata o aumento do comprimento do cateter para que ele aumente a resistência e a passagem do liquor na medida exata da resistência fisiológica.

Numa publicação anterior, ${ }^{2}$ também foram estudadas as características do fluxo e resistência de modelos de vários tipos de cateteres peritoneais comerciais isoladamente; adotando o conceito de Kajimoto et al. ${ }^{16}$ estimamos quais seriam as alterações nas fendas do cateter peritoneal para que houvesse uma compensação no efeito sifão próxima ao ideal.

Entretanto, nenhum trabalho até o momento avaliou os efeitos do cateter peritoneal no sistema valvular como um todo. Válvulas são produzidas de acordo com normas internacionais (ISO 7197), e essas normas determinam que a DVP é composta de três partes distintas, ou seja, um cateter proximal, que dá acesso ao sistema liquórico, a válvula, que impõe uma resistência adicional ao fluxo liquórico, e o cateter distal. Esses componentes sintetizam a PF no conceito descrito por Fox et al. ${ }^{13,14} \mathrm{e}$ esses componentes funcionam fisicamente como um conjunto de resistências em série, obedecendo aos princípios de Bernoulli e à lei de Poiseuille, e cada componente opõe alguma resistência ao fluxo liquórico, e a pressão da válvula, expressão conhecida pelo neurocirurgião, é a resultante (ou somatório) das resistências (R) de todos os componentes do sistema valvular. A resistência $(R)$ é uma função influenciada por vários fatores. A fórmula matemática que melhor expressa a resistência $(\mathrm{R})$ ao fluxo através de um tubo, assumindo-se um fluxo laminar, é a lei de Poiseuille:

$$
\mathrm{R}=8 \eta \cdot \mathrm{L} / \pi \cdot \mathrm{r}^{4} \text { equação }
$$

em que $\eta=$ viscosidade (em centipoise), $\mathrm{L}=$ comprimento do tubo (em $\mathrm{mm}$ ), $\mathrm{r}=$ raio da luz do tubo. Assim, o comprimento do tubo (L) e a viscosidade do fluido no tubo $(\eta)$ influenciam diretamente a resistência, enquanto o raio do tubo influi na razão inversa à quarta potência. Ora, o fluxo (F) em um tubo está ligado à resistência $(\mathrm{R})$ por meio da relação:

$$
\mathrm{F}=\Delta \mathrm{P} / \mathrm{R} \text { equação. }
$$

Portanto, trocas de peças do sistema valvular que não respeitem os dimensionamentos originais podem causar alterações no regime hidráulico ao qual o paciente já se adaptou, podendo ser a causa de descompensações da hidrocefalia numa fase posterior à revisão valvular (hiper ou hipodrenagem). Este artigo apresenta o impacto hidrodinâmico quando ocorrem alterações nos dimensionamentos originais. Na figura 2 é possível visualizar como diminuições quase imperceptíveis no diâmetro do cateter peritoneal alteraram o perfil hidrodinâmico do sistema valvular testado. A partir dos testes com os componentes originais (Figura 2A), alterações de apenas $0,1 \mathrm{~mm}$ no diâmetro de um cateter peritoneal (Figura 2C) aumentou o efeito resistivo do sistema valvular suficientemente para que ele funcionasse totalmente fora do perfil hidrodinâmico preconizado originalmente; o efeito resistivo se acentuou (Figura 2D) quando trocamos o diâmetro do cateter peritoneal por um $0,2 \mathrm{~mm}$ menor; o efeito resistivo se amenizou (Figura 2B) quando o diâmetro do cateter peritoneal foi aumentado para $1,5 \mathrm{~mm}$, ou seja, $0,3 \mathrm{~mm}$ maior do que o cateter original, portanto diminuindo o efeito resistivo de todo o sistema valvular.

Portanto, alterações aparentemente insignificantes no diâmetro do tubo causaram importante repercussão na hidrodinâmica do sistema valvular, pois existe uma relação física exponencial (à quarta potência).

Da mesma maneira, alterações no comprimento do cateter também influenciaram no desempenho hidráulico da válvula, como podemos observar na tabela 2 e figura 3; a diminuição do comprimento do cateter alterou o perfil hidrodinâmico do sistema valvular de um sistema de regime de alta pressão para um regime de média pressão. As implicações clínicas são muito claras, tanto nas revisões de válvulas quanto nos implantes iniciais. Se o neurocirurgião cortar o cateter no momento do implante, e dependendo do comprimento cortado, a válvula trabalhará em regime hidráulico fora 
do especificado, e o raciocínio lógico do neurocirurgião também se torna comprometido. Esse fato pode ser crítico principalmente em crianças e neonatos, pois são procedimentos em que comumente grandes porções do cateter peritoneal são cortadas.

\section{Conclusões}

O cateter peritoneal tem sido gradativa e imperceptivelmente alterado ao longo da última década pela indústria, e a maioria dos fabricantes aumentou o tamanho do cateter e/ou diminuiu o diâmetro interno do tubo numa tentativa de amenizar o efeito sifão.

O objetivo deste estudo foi o de explorar os dimensionamentos do cateter peritoneal e observar os seus efeitos no comportamento hidráulico de todo o sistema valvular. Este trabalho demonstra claramente que a substituição do cateter peritoneal original em sistemas valvulares por outro com aspectos diferentes traz complicações para o paciente e desorienta o julgamento clínico do neurocirurgião no período pós-implante. Nas situações em que os neurocirurgiões encontrem a necessidade de troca do cateter, por motivos rotineiros, essa substituição deve ser por outro que apresente diâmetro e comprimento iguais aos do utilizado originalmente. $\mathrm{O}$ impacto de cortes de cateteres em sistemas valvulares de menor regime pressórico será avaliado posteriormente.

\section{Referências}

1. Maset AL, Camilo Pinto JR, Andrade JR, Xavier VEF. Considerações hidrodinâmicas sobre a derivação liquórica. Parte IV: Tecnologia de válvulas: $1^{\text {a }}$ Geração. Arq Bras Neurocir. 2009;28(3):87-96.

2. Maset AL, Castro SC, Camilo JR. Considerações hidrodinâmicas sobre a derivação liquórica. Parte I: Efeitos do cateter peritoneal. Arq Bras Neurocir. 2005;24(1):9-16.

3. Camilo Pinto JR. Simulação hidrodinâmica e caracterização experimental de mecanismos anti-sifão em sistemas de drenagem externa de líquido cefalorraquidiano [dissertação]. Ilha Solteira (SP): Universidade Estadual Paulista; 2005.

4. Camilo Pinto JR, Maset AL, Duarte KP, Mansur SS, Vieira EDR. An apparatus for testing external shunts utilized in hydrocephalus surgery treatment. In: $11^{\circ}$ Congress of Thermal Sciences and Engineering, Curitiba; 2006.
5. Camilo Pinto JR, Maset AL, Duarte KP, Vieira DDR, Perini EA, Vieira EDR. Mechanical tests of tree-way valves of external neurosurgical shunts. In: Brazilian Conference on Dynamics, control and their Applications - DINCON, 5. Guaratinguetá; 2006.

6. Camilo Pinto JR, Maset AL, Mansur SS, Vieira EDR. Bancada para simulação hidrodinâmica de mecanismos anti-sifão em sistemas de drenagem externa do líquido cefalorraquidiano. In: IV Congresso Temático de Dinâmica, Controle e Aplicações, Bauru, SP; 2005.

7. Maset AL, Camilo JR. O efeito sifão em pacientes com drenagem externa. In: VI Congresso da Sociedade Brasileira de Neurocirurgia Pediátrica. Belo Horizonte, 2005. Anais. Belo Horizonte; 2005.58 p. 48.

8. Maset AL, Camilo JR, Duarte KP, Vieira ER. Considerações hidrodinâmicas sobre a derivação liquórica. Parte III: Distúrbios hidrodinâmicos em sistemas de drenagem externa. Proposta de solução. Arq Bras Neurocir. 2006;25(3):100-11.

9. Maset AL, Camilo Pinto JR, Vieira DDR. Considerações hidrodinâmicas sobre a derivação liquórica. Parte II: O efeito sifão em sistemas de drenagem externa. Arq Bras Neurocir. 2005;24(2):45-51.

10. Maset AL, Camilo JR, Vieira EDR. CSF dynamics in external drainage systems: the siphoning effect and possible role of anti-siphon devices. In: Hydrocephalus 2006, Proceedings Goteborg Sweden; 2006.

11. McCullough DC. Symptomatic progressive ventriculomegaly in hydrocephalics with patent shunts and antisiphon devices. Neurosurgery. 1986;19(4):617-21.

12. Pudenz RH, Foltz EL. Hydrocephalus: overdrainage by ventricular shunts. A review and recommendations. Surg Neurol. 1991;35(3):200-12.

13. Fox JL, McCullough DC, Green RC. Effect of cerebrospinal fluid shunts on intracranial pressure and on cerebrospinal fluid dynamics. 2. A new technique of pressure measurements: results and concepts. 3 . A concept of hydrocephalus. J Neurol Neurosurg Psychiatry. 1973;36(2):302-12.

14. Fox JL, McCullough DC, Green RC. Cerebrospinal fluid shunts: an experimental comparison of flow rates and pressure values in various commercial systems. J Neurosurg. 1972;37(6):700-5.

15. de Jong DA, Delwel EJ, Avezaat CJ. Hydrostatic and hydrodynamic considerations in shunted normal pressure hydrocephalus. Acta Neurochir (Wien). 2000;142(3):241-7.

16. Kajimoto $\mathrm{Y}$, Ohta $\mathrm{T}$, Miyake $\mathrm{H}$, Matsukawa M, Ogawa D, Nagao K, et al. Posture-related changes in the pressure environment of the ventriculoperitoneal shunt system. J Neurosurg. 2000;93(4):614-7.

17. Mukerji N, Cahill J, Rodrigues D, Prakash S, Strachan R. Flow dynamics in lumboperitoneal shunts and their implications in vivo. J Neurosurg. 2009;111(3):632-7.

\section{Endereço para correspondência}

Angelo Luiz Maset

Av. Dr. Carlos de Arnaldo, 360, Jd. Palmeiras

15093-000 - Condomínio Village Sta. Helena,

São José do Rio Preto, SP, Brasil

E-mail: maset@terra.com.br 